Draft version August 9, 2021

Preprint typeset using $\mathrm{LAT}_{\mathrm{E}} \mathrm{X}$ style emulateapj v. 5/2/11

\title{
THE LONG-TERM POST-OUTBURST SPIN DOWN AND FLUX RELAXATION OF MAGNETAR SWIFT J1822.3-1606
}

\author{
P. Scholz ${ }^{1,2}$, V. M. KAspi ${ }^{1}$, And A. Cumming ${ }^{1}$ \\ Draft version August 9, 2021
}

\begin{abstract}
The magnetar Swift J1822.3-1606 entered an outburst phase in 2011 July. Previous X-ray studies of its post-outburst rotational evolution yielded inconsistent measurements of the spin-inferred magnetic field. Here we present the timing behavior and flux relaxation from over two years of Swift, RXTE, and Chandra observations following the outburst. We find that the ambiguity in previous timing solutions was due to enhanced spin down that resembles an exponential recovery following a glitch at the outburst onset. After fitting out the effects of the recovery, we measure a long-term spin-down rate of $\dot{\nu}=(-3.0 \pm 0.3) \times 10^{-8} \mathrm{~s}^{-2}$ which implies a dipolar magnetic field of $1.35 \times 10^{13} \mathrm{G}$, lower than all previous estimates for this source. We also consider the post-outburst flux evolution, and fit it with both empirical and crustal cooling models. We discuss the flux relaxation in the context of both crustal cooling and magnetospheric relaxation models.

Subject headings: pulsars: general — pulsars: individual (Swift J1822.3-1606) — stars: neutron — X-rays: stars
\end{abstract}

\section{INTRODUCTION}

Magnetars are neutron stars whose radiation is powered by the decay of their enormous magnetic fields $\left(10^{13-15} \mathrm{G}\right)$ rather than by rotation, as are the vast majority of non-accreting pulsars (for reviews, see Woods \& Thompson 2006; Mereghetti 2008). The large magnetic fields of magnetars cause extreme activity such as large outbursts during which their X-ray flux output can increase by several orders of magnitude, accompanied by short energetic X-ray bursts (for a review of magnetar activity see Rea \& Esposito 2011).

Nearly every magnetar outburst has been accompanied by a change in the timing properties of the pulsar (Dib \& Kaspi 2013). The most common timing change that is observed is a spin-up glitch contemporaneous with the outburst onset. Following a glitch, the spin period of a magnetar can exponentially recover, although sometimes only partially, to its pre-glitch value. In some cases the recovery has overcompensated for the spin-up glitch and the net effect is a spin down (Livingstone et al. 2010; Gavriil et al. 2011). In the cases where a spin-up glitch has not been observed, other timing changes and anomalies have been noted, such as enhanced spin down and an increase in timing noise (e.g. Dib et al. 2009, 2012).

The magnetar Swift J1822.3-1606, was discovered when an X-ray burst was detected by the Swift Burst Alert Telescope (BAT) on 2011 July 14 (Cummings et al. 2011). It was found to be a pulsar when an 8.43-s periodicity was identified using $R X T E$ (Göğüs et al. 2011). Livingstone et al. (2011) reported on initial timing and spectroscopic results from Swift, RXTE, and Chandra and showed that the flux of the source was decaying from its peak at the onset of the outburst. A spin-down rate of $\dot{P}=2.54 \times 10^{-13}$ was measured which implied a surface dipolar magnetic field of $B=4.7 \times 10^{13} \mathrm{G}$. The character-

\footnotetext{
${ }^{1}$ Department of Physics, Rutherford Physics Building, McGill University, 3600 University Street, Montreal, Quebec, H3A 2T8, Canada

2 pscholz@physics.mcgill.ca
}

istic post-outburst decay and high magnetic field value confirmed that Swift J1822.3-1606 was a new magnetar that had experienced an outburst on 2011 July 14.

Subsequent studies of the post-outburst timing evolution of Swift J1822.3-1606 found that the spin-inferred magnetic field may be lower than that measured in Livingstone et al. (2011). Rea et al. (2012) presented a timing solution in which the spin-inferred magnetic field was measured to be $2.7 \times 10^{13} \mathrm{G}$. Scholz et al. (2012) fit several timing solutions to the post-outburst Swift, $R X T E$, and Chandra observations and found that a model with only non-zero $P$ and $\dot{P}$ (similar to what was presented in Rea et al. 2012) was not a good fit to the data and that the addition of higher period derivatives improved the fit. Their best fit utilized three significant period derivatives and the measured $\dot{P}$ in that case implied a spin-inferred B-field of $\sim 5 \times 10^{13} \mathrm{G}$. There has thus been some ambiguity in the best timing solution, and parameters, like $B$, derived from those solutions for Swift J1822.3-1606.

The flux and spectral evolution of Swift J1822.3-1606 was also presented in Rea et al. (2012) and Scholz et al. (2012). They found that the flux decayed rapidly following the outburst and that the spectral parameters softened. This behavior followed the hardnessflux correlation that is generally expected in the twisted-magnetosphere model (Thompson et al. 2002; Lyutikov \& Gavriil 2006; Beloborodov 2009) and observed in many other magnetars (e.g. Israel et al. 2007; Scholz \& Kaspi 2011). On the other hand, it was found that a model of the thermal relaxation of the magnetar crust reproduced the observed luminosity decay well. Both Rea et al. (2012) and Scholz et al. (2012) reported that, in these models, the late time decay was not well constrained and depended on parameters of the inner crust. X-ray flux observations at late times, when the source is closer to quiescence, could better constrain the models and thus implied parameters of the neutron star crust in this picture. The magnetospheric twist model 
also makes specific predictions for the flux and spectral evolution. In particular the twist relaxation time scale depends in a well defined way on the stellar magnetic moment, the electric voltage sustaining magnetospheric discharge, as well as on the emitting area, and can be tested if these parameters are constrained (Beloborodov 2009). Also, the thermal luminosity is predicted to vary as the emitting area squared, which can also be tested.

In this paper we present updated timing solutions for Swift J1822.3-1606 with a baseline that is over twice as long as in Scholz et al. (2012), using new data from the Swift X-ray Telescope (XRT). From this we attempt to resolve the previous ambiguity regarding the true value of the spin-down rate and hence the magnetic field. We also present the up-to-date flux and spectral evolution. We discuss how the timing and spectral evolutions have changed since previous studies and the implications of the new results in the context of the magnetar model.

\section{OBSERVATIONS}

\subsection{Swift Observations}

Since the 2011 July 14 (MJD 55756) outburst of Swift J1822.3-1606, Swift/XRT (Burrows et al. 2005) has been used to obtain 61 observations of the source for a total exposure time of $297 \mathrm{ks}$. The exposure times for each individual observation ranged from 0.5 to $18 \mathrm{ks}$. Data were collected in two different modes, Photon Counting (PC) and Windowed Timing (WT). While the former gives full imaging capability with a time resolution of $2.5 \mathrm{~s}$, the latter forgoes imaging to provide $1.76-\mathrm{ms}$ time resolution by reading out events in a collapsed onedimensional strip. As the PC mode time resolution is insufficient for our timing analysis, those data are used only for our spectral work.

For each observation, the unfiltered Level 1 data were downloaded from the Swift quicklook archive 3 . The standard XRT data reduction script, xrtpipeline, was then run using the source position of $\mathrm{RA}=18^{\mathrm{h}} 22^{\mathrm{m}} 18^{\mathrm{s}}$, Dec $=-16^{\circ} 04^{\prime} 26^{\prime \prime} .8$ (Pagani et al. 2011) and the best available spacecraft attitude file. Events were then reduced to the solar-system barycenter using the above source position. Source and background events were extracted using the following regions: for WT mode, a 30-pixel long strip centered on the source was used to extract the source events and a 50-pixel long strip positioned away from the source was used to extract the background events. For PC mode, a circular region with radius 20 pixels was used for the source region and an annulus with inner radius 40 pixels and outer radius 60 pixels was used as the background region. For the first (00032033001) and second (00032033017) PC mode observation, circular regions with radii of 6 and 2 pixels, respectively, were excluded to avoid pileup.

For WT mode data, exposure maps, spectra, and ancillary response files were created for each individual orbit. The spectra and ancillary response files were then summed to create a spectrum for each observation. For the PC mode data, exposure maps, spectra and ancillary response files were created on a per observation basis. We used response files for spectral fitting from the 20130313 CALDB and version 6.13 of HEASOFT. In Swift/XRT

\footnotetext{
${ }^{3}$ http://swift.gsfc.nasa.gov/cgi-bin/sdc/ql
}

observations, there are columns of bad pixels that can disrupt the source PSF. Orbits were not used in an observation if the bad columns were found to be within 3 pixels of the source position.

\subsection{RXTE and Chandra Observations}

We downloaded 32 RXTE observations from the $H E A S A R C$ archive. These data spanned the MJD range from 55758 to 55893 (2011 July 16 to 2011 Nov 28), for a total of $174 \mathrm{ks}$ of integration time. The data were collected in GoodXenon mode which records each event with $1-\mu$ s time resolution.

Following the outburst, our ToO program with the Chandra X-ray Observatory was triggered. Five ACIS Continuous Clocking (CC) mode observations were obtained between MJD 55769 and 56036 (2011 July 27 and 2012 April 19), with exposures ranging from 10 to $20 \mathrm{ks}$. CC-mode has a time resolution of $2.85 \mathrm{~ms}$ and sensitivity between 0.3 and $10 \mathrm{keV}$.

The RXTE and Chandra sets of observations are identical to those used in Scholz et al. (2012) and are summarized in Table 1 of that work.

\section{ANALYSIS \& RESULTS}

\subsection{Timing}

For each Swift and Chandra observation, a pulse time of arrival was extracted using the maximum likelihood method described in Scholz et al. (2012). RXTE TOAs were measured using cross-correlation with a template profile, as the maximum likelihood method is computationally too expensive for these data due to the high number of counts. We then fit timing solutions to the TOAs using the TEMPD5 pulsar timing software package.

We first fit a timing solution that included only a frequency and frequency derivative as was done by Rea et al. (2012) and for Solution 1 of Scholz et al. (2012). This model did not fit the data well, with reduced $\chi_{\nu}^{2}=7.38$ for 83 degrees of freedom. In order to be sensitive to only the long-term spin down of the pulsar, we then fit the same model to all TOAs from observations taken later than approximately two months (MJD $\geq 55800$ ) from the onset of the outburst. We found that this provided an acceptable fit $\left(\chi_{\nu}^{2} / \nu=1.19 / 41\right)$, and that it was much improved from the $\nu$ and $\dot{\nu}$ fit to the entire data set. We noted that the excluded TOAs appeared to form an exponential decay in the phase residuals (top-panel of Figure1). We therefore added an exponential glitch recovery to our model with the glitch epoch at the time of the Swift/BAT burst trigger (MJD 55756). The glitch recovery model provided an excellent fit with $\chi_{\nu}^{2} / \nu=0.97 / 81$. Table 1 shows the results of this fit and the bottom panel of Figure 1 shows the best-fit residuals.

In addition to the exponential recovery model, we tried some alternative models. We attempted to fit the residuals with higher derivatives similar to Scholz et al. (2012). We found that in order to produce a fit with a similar $\chi_{\nu}^{2}$, five frequency derivatives were needed. However, since the long-term spin down is well fit by the simpler $\nu$ and $\dot{\nu}$ timing solution, and extra derivatives are not needed if the first two months following the outburst is ignored, it

\footnotetext{
4 http://cxc.harvard.edu/proposer/POG/html/

5 http://tempo.sourceforge.net
} 
is clear that the multi-derivative solution is not representative of the true spin down of the pulsar and was only an artifact due to the contamination of the enhanced spin down at early times. We also compared the two models by fitting them to all of the data excluding the last three months and seeing how well they predicted the excluded TOAs. The single-derivative glitch recovery model predicted the last three months within $5 \%$ in phase and the measured parameters were fully consistent with the fit to the whole data set. The multi-derivative model, however, did not predict the later data with the last three months of TOAs wandering up to $30 \%$ in phase.

We also attempted to fit the post-outburst TOAs with a change in $\nu$ at MJD 55900 as the timing residuals indicated a deviation in the rotation from the long-term spin down prior to that epoch. This did not provide an acceptable fit, so we also tried a change in $\dot{\nu}$. This gave a slightly higher $\chi_{\nu}^{2} / \nu(1.22 / 81)$ than in the exponential recovery model. Aside from the poorer fit, this latter solution seems contrived given the absence of precedence for such behavior post-outburst in magnetars. As discussed in Section 4.1, there are many examples of glitches accompanied by exponential recoveries in magnetar outbursts. For these and the aforementioned reasons, we conclude that the glitch recovery model is by far the most likely.

Although we model the post-glitch spin down with a glitch recovery, we cannot conclusively say that a glitch occurred. This is because timing observations of the source pre-outburst are not available. However, if the pre-detection spin-down frequency was the same as its long-term post-outburst value (i.e. the exponential recovery perfectly compensated for the spin-up glitch), the fractional magnitude of the hypothetical glitch would be $\Delta \nu / \nu=(2.3 \pm 0.1) \times 10^{-7}$ which is in the typical range of glitch magnitudes observed from magnetars $\left(\Delta \nu / \nu \sim 10^{-7}-10^{-5}\right.$; e.g. Dib \& Kaspi 2013). An under-recovery wound imply a larger glitch, whereas an over-recovery a smaller glitch.

\subsection{Flux and Spectral Evolution}

We fit a photoelectrically absorbed blackbody plus power-law model to each Swift and Chandra spectrum using XSPEC6 v12.8. The hydrogen column density, $N_{\mathrm{H}}$, was fixed to the value measured by Scholz et al. (2012), $4.53 \times 10^{21} \mathrm{~cm}^{-2}$, and we used the XSPEC phabs model with abundances from Anders \& Grevesse (1989) and photoelectric cross sections from Bałucińska-Church \& McCammon (1992). For observations later than MJD 55975, sets of observations nearby in time were fit with joint $k T$ and $\Gamma$. This was done as the spectral parameters were not well constrained for individual observations and for each set $k T$ and $\Gamma$ were consistent from observation to observation. The flux was left free to vary from observation to observation as significant flux evolution was still present. The reduced $\chi_{\nu}^{2}$ values from the spectral fits ranged from 0.81 to 1.5 .

Figure 2 shows the results of the spectral fits. The flux is seen to decay following the outburst and the $k T$ and $\Gamma$ spectral parameters soften. The blackbody temperature, $k T$, remained approximately constant at $\sim 0.75 \mathrm{keV}$, or

\footnotetext{
6 http://xspec.gfsc.nasa.gov
}

perhaps even increased, in the first 10 days following the outburst onset at the BAT trigger. It then decreased to $\sim 0.6 \mathrm{keV}$ between 10-100 days from the trigger. Since MJD 55900, the blackbody temperature has remained roughly constant. The photon-index, $\Gamma$ appears to have increased (softened) following the outburst. This is most evident from the Chandra observations where the photon-index softened from $\sim 2.0$ to $\sim 2.5$ between 10 and 300 days from the trigger.

In order to characterize the flux relaxation we fit exponential decay models to the fluxes measured from the X-ray spectra. We first attempted a double-exponential decay model, $F(t)=F_{1} \exp ^{-\left(t-t_{0}\right) / \tau_{1}}+F_{2} \exp ^{-\left(t-t_{0}\right) / \tau_{2}}+F_{q}$. This did not provide an acceptable fit, as the reduced $\chi_{\nu}^{2} / \nu$ was $2.26 / 56$. So, we then fitted a triple exponential model: $F(t)=$ $F_{1} \exp ^{-\left(t-t_{0}\right) / \tau_{1}}+F_{2} \exp ^{-\left(t-t_{0}\right) / \tau_{2}}+F_{3} \exp ^{-\left(t-t_{0}\right) / \tau_{3}}+F_{q}$ In both cases, $F_{q}$ is the quiescent $1-10 \mathrm{keV}$ flux, $3 \times 10^{-14} \mathrm{erg} \mathrm{cm}^{-2} \mathrm{~s}^{-1}$, implied from the $0.2-2.4 \mathrm{keV}$ flux and spectral model measured from a 1993 ROSAT observation (Scholz et al. 2012). The triple exponential model provides a much better, and acceptable, fit with $\chi_{\nu}^{2} / \nu=0.95 / 54$. The best-fit exponential decay timescales were $6 \pm 1,27 \pm 2$, and $320 \pm 20$ days.

\section{DISCUSSION}

We have presented over a year of new Swift/XRT observations of Swift J1822.3-1606 which have increased the baseline to over two and a half years. This has allowed us to better measure the timing and spectral evolution. Importantly, we have presented a more straightforward timing model than that in Scholz et al. (2012) as well as evidence for a glitch at the epoch of the outburst onset. Our new timing model implies a long-term spin-down rate that is significantly smaller than previous estimates. We have also updated the post-outburst flux and spectral evolution of Swift J1822.3-1606 and show that it is well fit by a triple-exponential model that is decaying to the ROSAT measured quiescent flux. Below we discuss the implications of these findings.

\subsection{Post-outburst spin-down behavior}

Previous studies of the timing evolution of Swift J1822.3-1606 did not find a consistent timing solution. Livingstone et al. (2011) first presented a timing solution with just $\nu$ and $\dot{\nu}$ fit to the first $\sim 80$ days following the outburst and measured $B=4.7 \times 10^{13} \mathrm{G}$. This was followed by Rea et al. (2012) who, with 275 days of observations, found a lower value of $B=2.7 \times 10^{13} \mathrm{G}$. Both studies found that there was an unmodelled trend in their residuals, possibly attributed to timing noise. Scholz et al. (2012) attempted to fit the trend with higher frequency derivatives. They found that as higher derivatives were added, the value of $B$ increased. The best fit was found with three frequency derivatives (Solution 3 ) and $B=5.1 \times 10^{13} \mathrm{G}$, larger than the Rea et al. (2012) estimate.

Here we find that in the initial $\sim 100$ days following the onset of the outburst, the timing behavior of Swift J1822.3-1606 was not representative of the longterm spin down of the pulsar. Specifically, it was spinning down more rapidly, likely due to a recovery from a 
glitch. By using the data from the initial post-outburst epochs, the previous studies measured higher spin-down rates because of contamination from the early enhanced spin down. This is shown by the fact, pointed out by Tong \& Xu (2013), that as the solutions were derived from longer timing baselines, the measured spin-down rate became lower.

Glitches in pulsars and magnetars have been known to show exponential recoveries. Magnetars 1RXS J170849.0-400910 and 1E 2259+586 showed clear exponential recoveries following their 2001 and 2002 glitches (Kaspi \& Gavriil 2003; Woods et al. 2004) and $4 \mathrm{U} \quad 0142+61$ showed a slight over-recovery following its 2006 glitch (Gavriil et al. 2011). The magnetically active rotation-powered pulsar PSR J1846-0258 showed a spin-up glitch with a large over-recovery (Livingstone et al. 2010). The decay timescale measured for Swift J1822.3-1606 (40 days; see Table 11) is comparable to the analogous timescale for the 2001 glitch of 1RXS J170849.0-400910 (43 days) and somewhat longer than was observed in $1 \mathrm{E} 2259+586$ (17 days) and 4U 0142+61 (12-17 days; Dib \& Kaspi 2013). We note that in the single well sampled anti-glitch, during the 2012 outburst of magnetar 1E 2259+586, no exponential recovery was seen (Archibald et al. 2013). Without knowledge of the pre-outburst spin down of Swift J1822.3-1606, we cannot conclusively argue for the occurrence of a glitch, but given the exponential form of the post-outburst spin down and the prominence of exponential glitch recoveries in other magnetars, it seems extremely plausible.

However, the occurrence of enhanced spin down without the occurrence of a glitch following a magnetar outburst has also been observed. Following the the 2002 outburst of 1E 1048.1-5937 no glitch was observed and the magnitude of $\dot{\nu}$ increased as the pulsed flux decreased (Dib et al. 2009). The magnetars 1E 1547.0-5408 and SGR 1745-2900 showed similar behavior after their 2008 and 2013 outbursts, respectively (Dib et al. 2012; Kaspi et al. 2013). The same behavior is clearly not present in the post-outburst timing of Swift J1822.3-1606. In its case, as the flux of the magnetar decreased, the magnitude of $\dot{\nu}$ decayed. Thus, the post-outburst timing behavior of Swift J1822.3-1606 looks like a glitch recovery as observed from 1RXS J170849.0-400910, 1E 2259+586, and $4 \mathrm{U} 0142+61$ and does not resemble the more unusual enhanced spin-down behavior seen in 1E 1048.1-5937 and 1E $1547.0-5408$.

The enhanced spin-down rate following the outburst of Swift J1822.3-1606 was a factor of several larger than its long-term spin-down rate. The instantaneous post-glitch spin-down rate at the glitch epoch for an exponential recovery can be quantified by $\Delta \nu_{d} / \tau$ (Dib et al. 2008). For Swift J1822.3-1606, this quantity is $\Delta \nu_{d} / \tau=(26 \pm 5) \dot{\nu}$ where $\dot{\nu}$ is the value we report in Table 1 . This is higher than in previous magnetar glitches, the next highest being the 2002 glitch of $1 \mathrm{E} 2259+586$ with a $\Delta \nu_{d} / \tau=$ $(8.2 \pm 0.6) \dot{\nu}$. For radio pulsars, the post-glitch recoveries usually result in spin-down enhancements of only a few percent (e.g. Flanagan 1990; Wong et al. 2001).

Since many magnetars are observed frequently after their outburst, but are not followed up with longterm timing campaigns as has been Swift J1822.3-1606, it is possible that the measurements of their spindown rates are not representative of their long-term timing evolution. For example, the magnetic field of CXOU J164710.2-455216 was measured to be $\sim 1 \times$ $10^{14} \mathrm{G}$ using phase-coherent timing in the first 100200 days following its 2006 outburst (Israel et al. 2007; Woods et al. 2011), but An et al. (2013) have recently placed an upper limit on the long-term spin-down rate and hence inferred magnetic field of $<7 \times 10^{13} \mathrm{G}$ using a timing baseline of $\sim 6$ years.

The magnetic field measured from the long-term spin down of Swift J1822.3-1606 is $1.35 \times 10^{13} \mathrm{G}$. This is the second lowest spin-down inferred magnetar dipolar magnetic field and is about a factor of two higher than the lowest measured value, $6.1 \times 10^{12} \mathrm{G}$ of SGR $0418+5729$ (Rea et al. 2013). However, since pulsar timing is only sensitive to the surface dipolar component of the field, the true magnetic field of the magnetar could be significantly higher than the value measured from pulsar timing if the toroidal component is much higher than the poloidal component or if the polodial field has significant multipolar contributions. Indeed, there is evidence for a much higher magnetic field for SGR $0418+5729$ from spectral modelling $\left(\sim 1 \times 10^{14} \mathrm{G}\right.$, Güver et al. 2011) and from a phase-resolved cyclotron absorption feature $\left(>2 \times 10^{14} \mathrm{G}\right.$, Tiengo et al. 2013).

\subsection{Models of flux relaxation}

In addition to the empirical model fits in Section 3.2, we fit the light curve with a model of crustal cooling (Cumming et al., in prep). In these models, the neutron star crust is heated by a sudden deposition of energy, and the subsequent thermal relaxation and cooling is followed by integrating the thermal diffusion equation in time. Both Scholz et al. (2012) and Rea et al. (2012) were able to fit the first 100 days of the light curve by depositing $\sim 10^{42} \mathrm{erg}$ in the outer crust at densities $\sim 10^{9}-10^{10} \mathrm{~g} \mathrm{~cm}^{-3}$. Scholz et al. (2012) found that at later times the model did not fit the data well, declining in flux more rapidly than observed. However, the late time lightcurve is sensitive to a number of physics inputs such as the neutron contribution to the heat capacity near neutron drip, thermal conductivity of the inner crust, and angular distribution of the heating around the star. We investigate this further here, updating our cooling models, and including the new flux measurements at times $\gtrsim 500$ days after outburst.

We have made two improvements to the model shown in Scholz et al. (2012). The first is in the treatment of the outer envelope. Rather than use the analytic flux-temperature relations for the outer envelope from Potekhin \& Yakovlev (2001), which are strictly correct only in the high density isothermal part of the crust, we have reproduced the detailed magnetized envelope models of Potekhin \& Yakovlev (2001), allowing us to match the envelope to the correct density at the top of our numerical grid (we typically follow the temperature on a grid in density extending from the crust/core boundary to $\approx 6 \times 10^{8} \mathrm{~g} \mathrm{~cm}^{-2}$ ). Because the temperature profile is not fully isothermal at these depths (deeper in the crust, the temperature profile is close to isothermal in steady-state because of the large thermal conductivity), using the analytic flux-temperature relation leads to a tens of percent underestimate of the flux and corre- 
sponding overestimate of the energy deposited. Second, we have improved our treatment of the magnetic field geometry. We calculate the cooling curve at the magnetic pole, where the magnetic field is radial, and then rescale that light curve appropriately for each local patch on the neutron star surface given the local magnetic field direction (a time-dependent extension of Greenstein \& Hartke $(1983))$. For heating across the whole surface and a dipole magnetic field, we find a more extended tail of the light curve at times of several hundred days and onwards compared to Scholz et al. (2012); the shape of the early time light curve does not change significantly. The late time tail comes from the equatorial region in which the field is close to horizontal, significantly reducing the radial thermal conductivity and increasing the cooling time.

Two models that reproduce the first 100 days of the light curve are shown in the second panel of Figure 2. We assume a distance of $1.6 \mathrm{kpc}$, neutron star mass $1.6 M_{\odot}$, radius $R=11.2 \mathrm{~km}$, core temperature $1.5 \times 10^{7} \mathrm{~K}$, and magnetic field strength at the pole $10^{14} \mathrm{G}$. The mass and radius correspond to the particular equation of state chosen by Brown \& Cumming (2009). As discussed in Scholz et al. (2012), a different choice of mass and radius primarily changes the cooling timescale by a factor of $1 / g^{2}$, where $g$ is the gravity, because the crust thickness is $\propto 1 / g$. The core temperature is chosen so that the luminosity at late times is $2 \times 10^{31} \mathrm{erg} \mathrm{s}^{-1}$, comparable to the ROSAT measurement of the quiescent flux. We then vary the energy injected and depth of heating to match the lightcurve. In the models shown in Figure 2, we deposit $\approx 3 \times 10^{42}$ erg in the outer crust down to a density of $\approx 10^{11} \mathrm{~g} \mathrm{~cm}^{-3}$, either over the whole surface assuming a dipole field geometry (as in Scholz et al. 2012), or over $15 \%$ of the neutron star surface at the magnetic pole. Both models predict the same flux evolution for times $\lesssim$ 100 days, but differ at late times. Heating a small region near the pole gives better agreement with the shape of the late time light curve. In this case the field is close to radial everywhere in the heated region, leading to more rapid cooling at late times which more closely matches the observed luminosity decrease.

Both models underpredict the luminosity at times $~$ 200 days by about a factor of two. As emphasized by Scholz et al. (2012) and Rea et al. (2012) there are several properties of the inner crust that can change the late time part of the light curve. A small amount of heat deposited in the inner crust can bring the late time lightcurve into agreement with the data. The shape of the decay at times $\gtrsim 200$ days is sensitive to the heat capacity of the layer of normal neutrons just below neutron drip. The thickness of this layer is determined by how quickly the critical temperature $T_{c}$ for superfluidity increases with density (Page \& Reddy 2012). We find that the best fits are obtained when we do not include the neutron heat capacity at all, i.e. $T_{c}$ rises very rapidly with density below neutron drip; a slow rise in $T_{c}$ leads to a slower decline in the light curve at late times. Another important parameter is the impurity parameter $Q_{\text {imp }}$ in the inner crust which determines the thermal conductivity. We set $Q_{\text {imp }} \approx 10$ in the models shown in Figure 2 .

It is rather remarkable that the models we have com- puted with standard assumptions for the inner crust physics reproduce the shape of the observed decay so well. It would be interesting to carry out a comprehensive survey of the parameters such as gravity, critical temperature for neutron superfluidity, inner crust thermal conductivity, magnetic field geometry and angular size of the heated region (e.g. see the similar study by Page \& Reddy 2013, for the accreting neutron star XTE J1701-462). The good agreement is especially interesting given the uncertainties in comparing the models with the data. These include the fact that the models predict the surface bolometric luminosity of the star, whereas the X-ray flux is measured in the narrow bandpass $1-10 \mathrm{keV}$. Similarly, the spectrum of the emission is not predominantly a thermal spectrum but instead is dominated by a power law component. Furthermore, the thermal part of the spectrum that is observed decreases in flux primarily due to a decreasing emitting area rather than a decreasing temperature as might be expected for a cooling surface. Explaining the observed decrease in emitting area is the major challenge for crust cooling models. Addressing the spectral evolution requires detailed modelling of the formation of the spectrum. Here we have assumed that while there can be significant modification of the spectrum, due to for example resonant Compton scattering in the magnetosphere, the luminosity remains largely unaffected, at least over the bulk of the evolution.

The magnetospheric untwisting model detailed by Beloborodov (2009) also makes specific predictions regarding the flux and spectral evolution of magnetars post-outburst. In this picture the post-outburst magnetosphere has been twisted due to crustal motions originating from stresses induced by the strong internal magnetic field. The twist is carried by a bundle of currentcarrying field lines (the "j-bundle") which is anchored in the crust on a footpoint of area $A$. This footpoint, bombarded by current particles, radiates thermal emission and both fades and shrinks as the j-bundle dissipates during relaxation and untwisting. The evolution time of the X-ray luminosity is predicted to be $t_{e v} \simeq 10^{7} \mu_{32} \Phi_{10}^{-1} A_{11.5} \mathrm{~s}$ (Beloborodov 2009; Mori et al. 2013), where $\mu_{32}$ is the magnetic moment in units of $10^{32} \mathrm{G} \mathrm{cm}{ }^{3}, \Phi_{10}^{-1}$ is the electric voltage sustaining $e^{ \pm}$ discharge in the magnetosphere in units of $10^{10} \mathrm{~V}$, and $A_{11.5}$ is the j-bundle footpoint area in units of $10^{11.5} \mathrm{~cm}^{2}$. For Swift J1822.3-1606, the relation predicts an evolution time scale of $\sim 10^{6} \mathrm{~s}$, or $\sim 10$ days assuming $\Phi_{1 \mathrm{n}}^{-1}=1$, reasonable given expectations (Beloborodov 2009). This is roughly consistent with the time scale ( $\sim 6$ days) we found for the fastest-decaying exponential component (see Section 3.2 ) but inconsistent with the second two time scales in the three-component decay model, unless $\Phi_{10}$ is significantly smaller than unity. It could be that the $\mathrm{j}$-bundle untwisting time scale corresponds to the shortest exponential decay, with overall crustal cooling corresponding to the latter two.

The untwisting model further predicts that the thermal X-ray luminosity from the heated footpoint should vary with $A^{2}$. Figure 3 shows the thermal X-ray flux of Swift J1822.3-1606 plotted against the emitting area as inferred from our blackbody fits. Here we compare the expectation of the magnetospheric twist model, 
namely $f \propto A^{2}$ with our data and find that this is not a good description of the data. Rather, our bestfit relation has $f \propto A^{3 / 2}$. The observed prefactor is larger than the model predicts even when the twist is maximal (of order unity) and the voltage drop is $\sim 10 \mathrm{GeV}\left(\Phi_{10} \approx 1\right)$. Beloborodov $(2009)$ predicts $L \approx$ $10^{34} \mathrm{erg} \mathrm{s}^{-1} B_{14} R_{6}^{-3} \psi \Phi_{10}\left(A / \mathrm{km}^{2}\right)^{2}$, where $\psi$ is the twist angle. The observed relation has $L=1.5 \times 10^{34} \mathrm{erg} \mathrm{s}^{-1}$ for $A=1 \mathrm{~km}^{2}$ and $d=1.6 \mathrm{kpc}$. However, $\Phi_{10}=1$ is on the upper end of the $0.1-1 \Phi_{10}$ range set by pair creation given by Beloborodov (2009), and the magnetic field we infer from spin down is significantly smaller than $10^{14} \mathrm{G}$, reducing the expected luminosity. In addition, the absorbed $1-10 \mathrm{keV}$ flux is an underestimate of the true bolometric flux.

The shallower scaling of luminosity with area could arise from a systematic change in the shape of the thermal spectrum compared to a blackbody as the flux decreases. Then the inferred area from blackbody fits would systematically change with flux, modifying the underlying $L \propto A^{2}$ scaling. An alternative physical explanation is that the field geometry is more complex than the dipole geometry assumed by Beloborodov (2009). The luminosity is $L \approx I \mathcal{V}$ where $I$ is the current, given from Ampère's law by $I \propto B_{\phi} a$ where $a$ is the radius of the flux bundle at the surface of the star. Following the bundle of field lines from one pole to the other through the magnetosphere (see Beloborodov 2009, Appendix A for a more detailed treatment), the twist angle is $\psi \approx\left(r_{\max } / a\right)\left(B_{\phi} / B_{P}\right)$, where $B_{P}$ is the poloidal field strength, $B_{\phi}$ the toroidal field in the twist, and $r_{\max }$ is the maximum radial extent of the flux tube. For a dipole field, $r_{\max } \approx R(R / a)^{2}$, giving $L \propto a^{4} \propto A^{2}$ as found by Beloborodov (2009). A different field geometry changes the scaling. For example, a quadrupole field has $r_{\max } \approx R(R / a)$, giving $L \propto a^{3} \propto A^{3 / 2}$, in agreement with the observed scaling in Figure 3. It may therefore be of interest to explore magnetospheric untwisting with a more complex field geometry.

\section{CONCLUSIONS}

We have presented an up-to-date analysis of the post-outburst flux and timing evolution for Swift J1822.3-1606. We find that the spin down following the outburst is well described with an exponential glitch recovery and that the long-term spin-down inferred magnetic field is lower than previously estimated. From this, we conclude that a glitch likely occurred near the outburst onset as has been seen in several other magnetar outbursts. We also find that the post-outburst flux evolution is consistent with thermal relaxation of the neutron star crust, particularly if heat was deposited internally in a small region close to neutron drip depth and near the magnetic axis. We find that flux relaxation due to magnetospheric untwisting may also be consistent if the poloidal magnetic field is more complicated than a simple dipole.

We are grateful to the Swift, Chandra, and RXTE teams for their flexibility in scheduling TOO observations. We thank Kostas Gourgouliatos for useful discus- sions. VMK holds the Lorne Trottier Chair in Astrophysics and Cosmology and a Canadian Research Chair in Observational Astrophysics and received additional support from NSERC via a Discovery Grant and Accelerator Supplement, by FQRNT via the Centre de Recherche Astrophysique de Quebec, and by the Canadian Institute for Advanced Research.

\section{REFERENCES}

An, H., Kaspi, V. M., Archibald, R., \& Cumming, A. 2013, ApJ, 763,82

Anders, E., \& Grevesse, N. 1989, Geochim. Cosmochim. Acta, 53, 197

Archibald, R. F., Kaspi, V. M., Ng, C.-Y., et al. 2013, Nature, 497, 591

Bałucińska-Church, M., \& McCammon, D. 1992, ApJ, 400, 699

Beloborodov, A. M. 2009, ApJ, 703, 1044

Brown, E. F., \& Cumming, A. 2009, ApJ, 698, 1020

Burrows, D. N., Hill, J. E., Nousek, J. A., et al. 2005, Space Sci. Rev., 120, 165

Cummings, J. R., Burrows, D., Campana, S., et al. 2011, The

Astronomer's Telegram, 3488

Dib, R., \& Kaspi, V. M. 2013, ApJ, submitted

Dib, R., Kaspi, V. M., \& Gavriil, F. P. 2008, ApJ, 673, 1044

-. 2009, ApJ, 702, 614

Dib, R., Kaspi, V. M., Scholz, P., \& Gavriil, F. P. 2012, ApJ, 748, 3

Flanagan, C. S. 1990, Nature, 345, 416

Gavriil, F. P., Dib, R., \& Kaspi, V. M. 2011, ApJ, 736, 138

Gögüus, E., Kouveliotou, C., \& Strohmayer, T. 2011, The

Astronomer's Telegram, 3491

Greenstein, G., \& Hartke, G. J. 1983, ApJ, 271, 283

Güver, T., Göğüş, E., \& Özel, F. 2011, MNRAS, 418, 2773

Israel, G. L., Campana, S., Dall'Osso, S., et al. 2007, ApJ, 664, 448

Kaspi, V. M., \& Gavriil, F. P. 2003, ApJ, 596, L71

Kaspi, V. M., Archibald, R. F., Bhalerao, V., et al. 2013, ApJ, submitted

Livingstone, M. A., Kaspi, V. M., \& Gavriil, F. P. 2010, ApJ, 710,1710

Livingstone, M. A., Scholz, P., Kaspi, V. M., Ng, C.-Y., \& Gavriil, F. P. 2011, ApJ, 743, L38

Lyutikov, M., \& Gavriil, F. P. 2006, MNRAS, 368, 690

Mereghetti, S. 2008, Astron. Astropys. Rev., 15, 225

Mori, K., Gotthelf, E. V., Zhang, S., et al. 2013, ApJ, 770, L23

Pagani, C., Beardmore, A. P., \& Kennea, J. A. 2011, The Astronomer's Telegram, 3493

Page, D., \& Reddy, S. 2012, ArXiv e-prints, arXiv:1201.5602

—. 2013, Phys. Rev. Lett., submitted; arXiv:1307.4455

Potekhin, A. Y., \& Yakovlev, D. G. 2001, A\&A, 374, 213

Rea, N., \& Esposito, P. 2011, in High-Energy Emission from Pulsars and their Systems, ed. D. F. Torres \& N. Rea (Springer ASSP), arXiv:1101.4472v1

Rea, N., Israel, G. L., Esposito, P., et al. 2012, ApJ, 754, 27

Rea, N., Israel, G. L., Pons, J. A., et al. 2013, ApJ, 770, 65

Scholz, P., \& Kaspi, V. M. 2011, ApJ, 739, 94

Scholz, P., Ng, C.-Y., Livingstone, M. A., et al. 2012, ApJ, 761, 66

Thompson, C., Lyutikov, M., \& Kulkarni, S. R. 2002, ApJ, 574, 332

Tiengo, A., Esposito, P., Mereghetti, S., et al. 2013, Nature, 500, 312

Tong, H., \& Xu, R.-X. 2013, Research in Astronomy and Astrophysics, 13, 1207

Wong, T., Backer, D. C., \& Lyne, A. 2001, ApJ, 548, 447

Woods, P. M., Kaspi, V. M., Gavriil, F. P., \& Airhart, C. 2011, ApJ, 726, 37

Woods, P. M., \& Thompson, C. 2006, in Compact Stellar X-ray Sources, ed. W. H. G. Lewin \& M. van der Klis (UK:

Cambridge University Press)

Woods, P. M., Kaspi, V. M., Thompson, C., et al. 2004, ApJ, 605, 378 
Table 1

Timing Parameters for Swift J1822.3-1606.

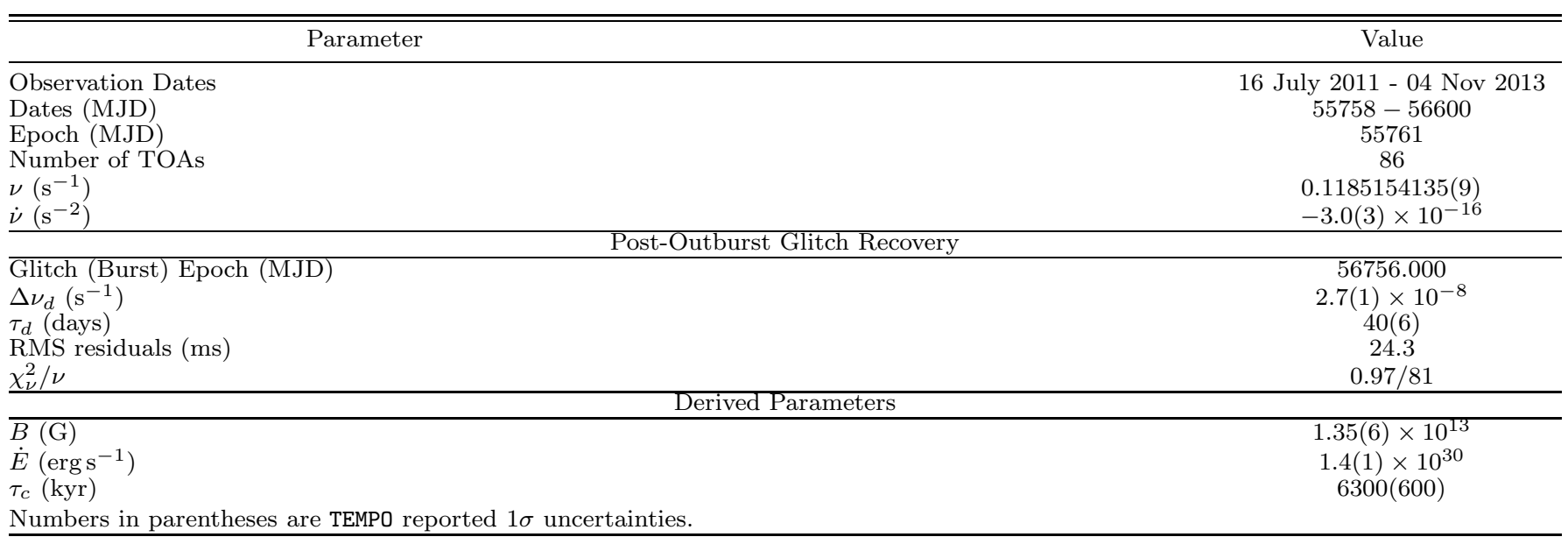

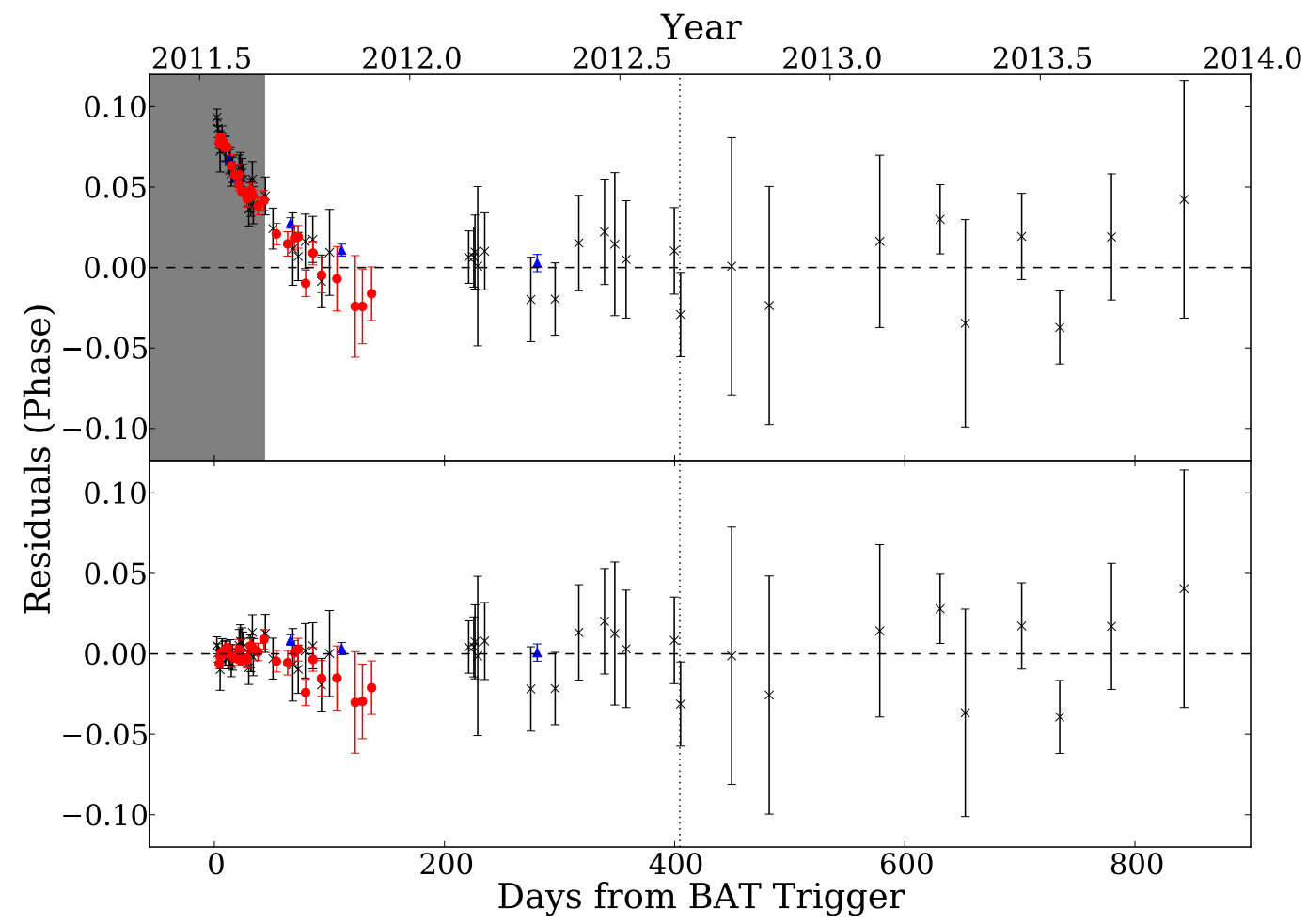

Figure 1. Timing residuals of Swift J1822.3-1606. The top panel shows the solution in Table 1 before the glitch recovery is fit. The bottom panel shows the residuals for the solution with the glitch recovery fit out. In both panels, black crosses represent Swift observations, red circles indicate $R X T E$ observations, and Chandra data are shown as blue triangles. The gray band represents the data that were excluded when the long-term spin down was fit prior to attempting to fit the glitch recovery. The vertical dotted line shows where the longest previous published timing solution ended (Scholz et al. 2012). 


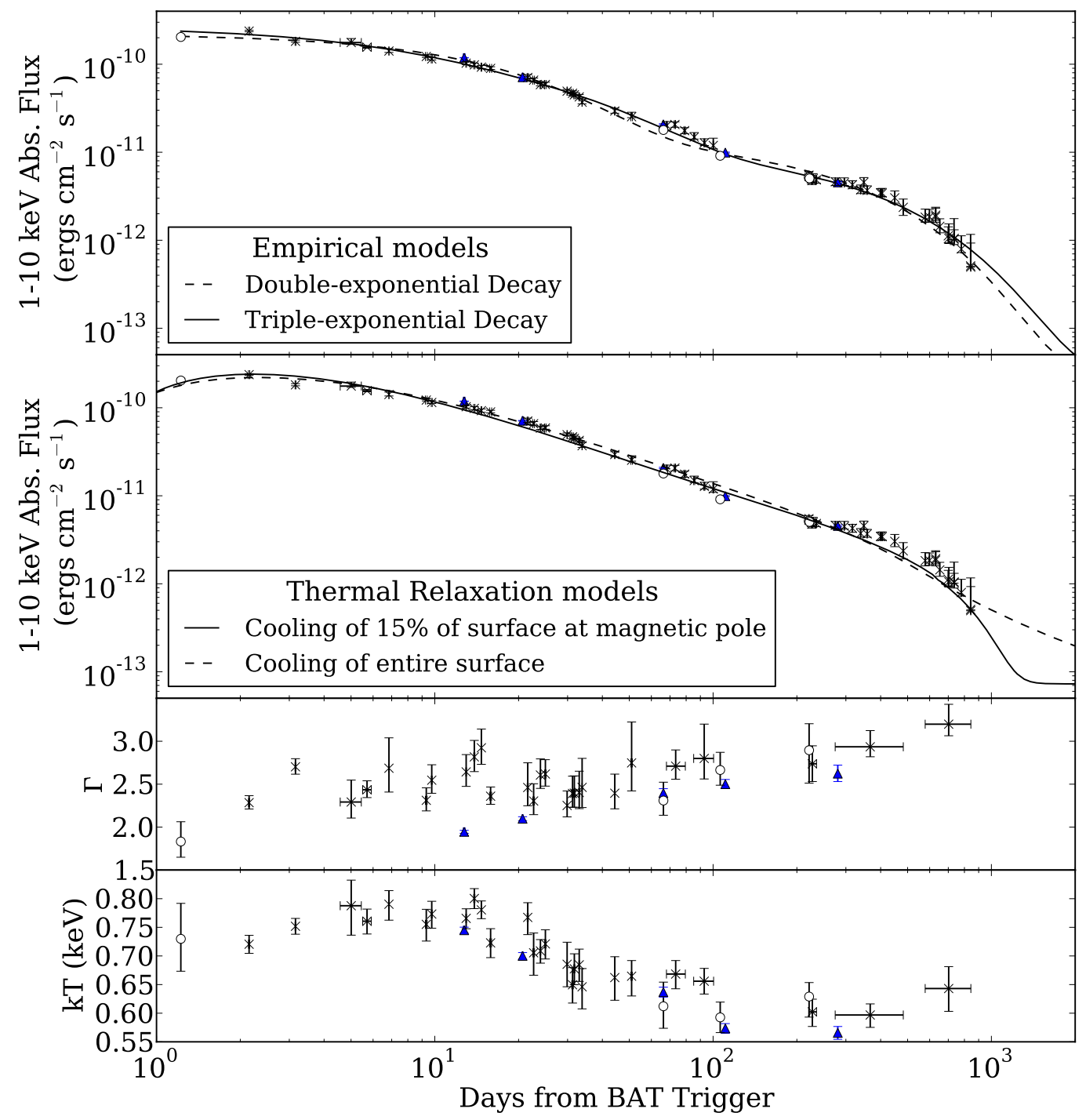

Figure 2. Spectral evolution of Swift J1822.3-1606. The top two panels both show the 1-10 keV flux evolution, but are fitted with two different sets of models. Black crosses denote Swift WT mode observations, open circles represent Swift PC mode data, and blue triangles show Chandra observations. In the spectral fits, for some observations $k T$ and $\Gamma$ are fit jointly in sets that are nearby in time, and so are represented by a single point in their respective plots. The horizontal error bars on $k T$ and $\Gamma$ respresent the extent in time of such sets. 


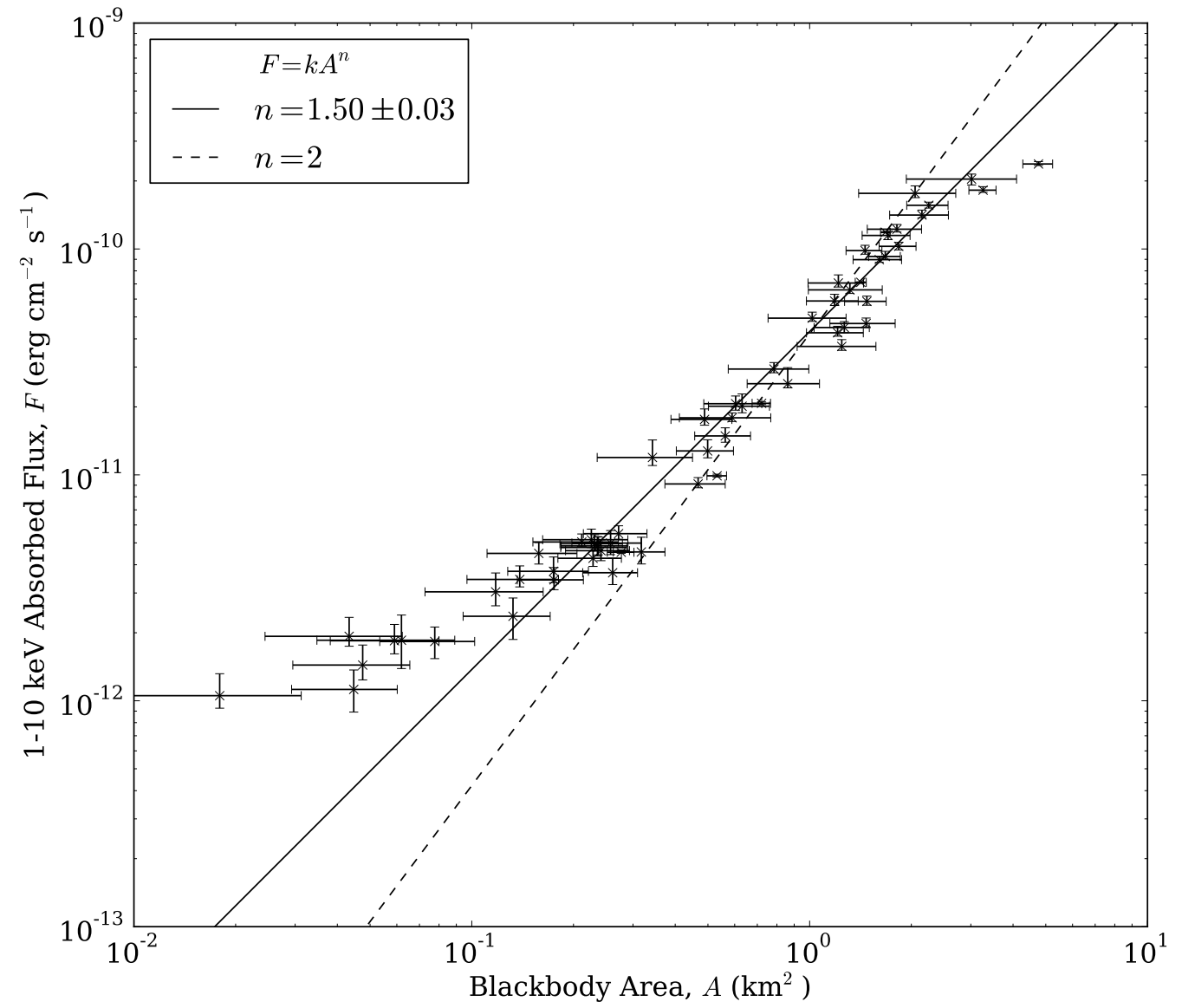

Figure 3. 1-10 keV absorbed flux as a function of the emitting area of the blackbody component of the spectral fits. To these data, we have fit the function $F=k A^{n}$. The solid line shows our best fit when allowing $n$ to vary, The best fit line in that case, in terms of luminosity (assuming a distance of $1.6 \mathrm{kpc}$ ), is $L=1.5 \times 10^{34} \mathrm{erg} \mathrm{s}^{-1}\left(A / 1 \mathrm{~km}^{2}\right)^{3 / 2}$. The dotted line shows the case $n=2$, as predicted by Beloborodov (2009). For that case, the best-fit line is given by $L=1.3 \times 10^{34} \mathrm{erg} \mathrm{s}^{-1}\left(A / 1 \mathrm{~km}^{2}\right)^{2}$. 